\title{
CT features of rupture of the thoracic aorta and subsequent death
}

\section{F van Gelderen $M D^{*}$}

\section{A M Packer $M D \mathrm{CM}^{* *}$}

* Department of Radiology,

** Department of Pathology, Wanganui Base Hospital, Wanganui, New Zealand
A

n 83-year-old man was found in a collapsed and dyspnoeic condition at his home and suffered a period of apnoea in transit to the hospital. On examination he was hypotensive with bradycardia and weak femoral pulses.

Chest radiographs revealed homogeneous opacification of the left hemithorax with relative sparing of the left base. The referring clinician considered the possibilities of rupture of the aorta or alternatively a mass in the left hemithorax; a left pleural tap revealed bloody fluid, and the patient was referred for computed tomography (CT).

A limited non-contrast-enhanced $\mathrm{CT}$ examination confirmed the diagnosis of aortic rupture. A dynamic contrast-enhanced examination demonstrated the signs to better advantage, with a large posterior 'encapsulated' extrapleural haematoma displacing the convex parietal pleura anteriorly. A smaller left anterior pleural fluid collection and marked mediastinal shift to the right side were present. The descending aorta was displaced anteriorly (Figure 1)

An initially confusing feature was the absence of contrast medium in the aorta, and lingering of contrast in the venae cavae and azygos venous system. There was also extensive dense opacification of dilated veins, including the spinal epidural veins lining the inside of the bony vertebral column, with loss of the normal rounded contour. Venous channels within the soft tissues posterior to the vertebral column, and contrast filled intercostal veins were also noted.

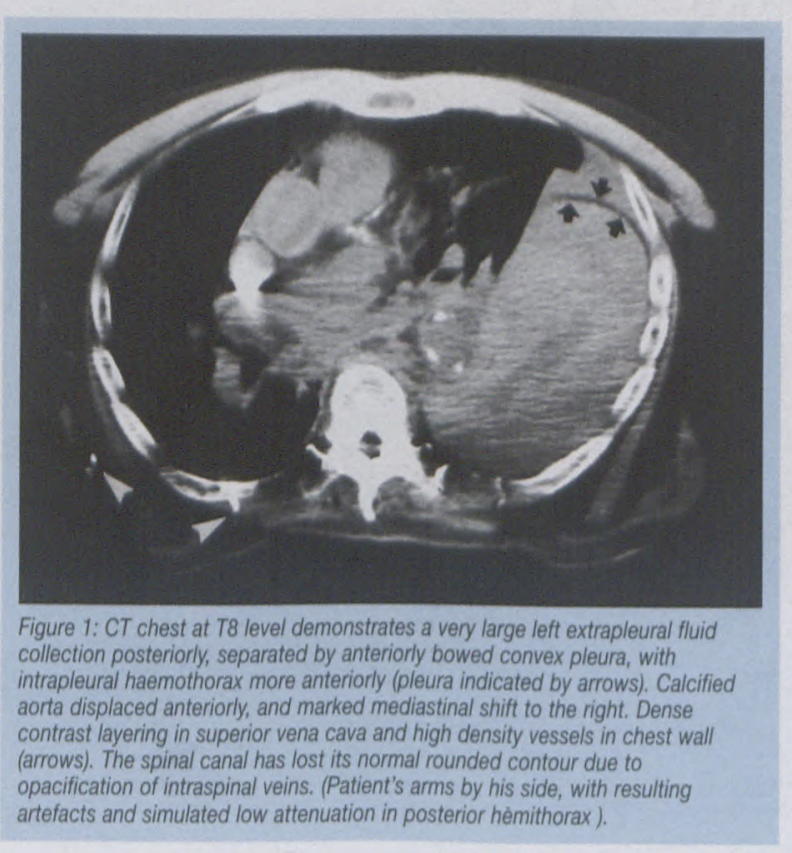

The dependent aspects of the hepatic veins (Figure 2), portal venous system (Figure 3) and right renal vein and radicles (Figure 4) were distended with contrast as if diagnostic venography had been performed. A posterior hepatic 'sinusoidogram' appearance was noted, with contrast readily filling the sinusoids in the dependent parts of the liver in a homogeneous 


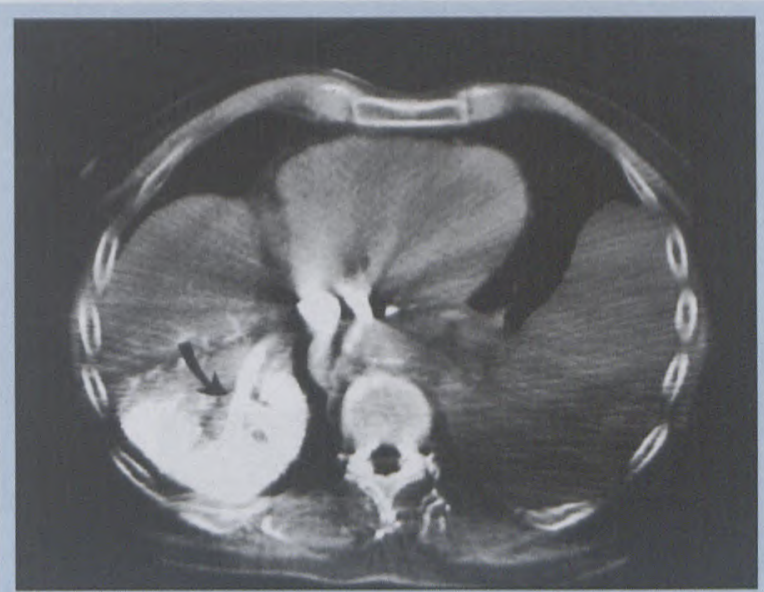

Figure 2: CT at T10-T11 disc level with very dense contrast medium in inferior vena cava (tip of nasogastric tube to the left of the inferior vena cava). Hepatic vein (arrow) and radicles distended with contrast medium, and sinusoids in dependent part of right lobe of liver homogeneously opacified. Note also opacification of venous channels related to posterior spinal elements at T10 level, within erector spinae muscles. The left extrapleural haematoma is again evident.

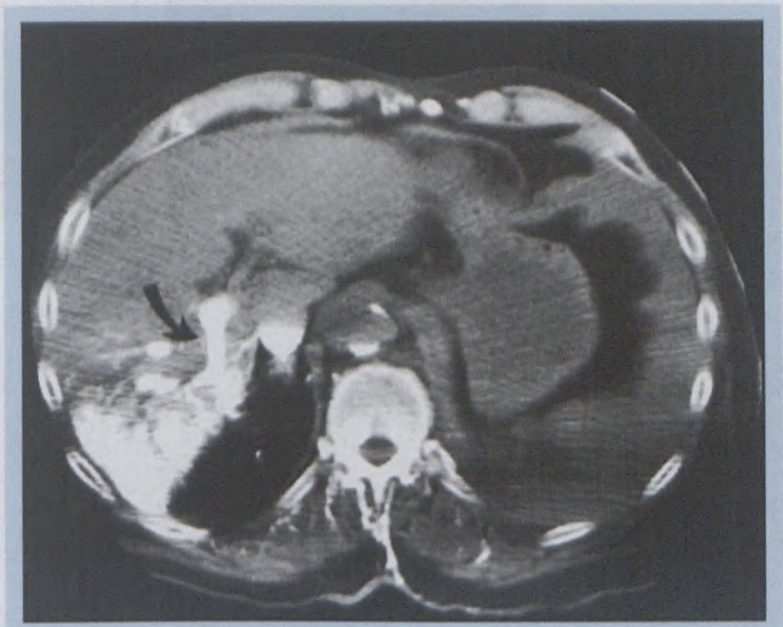

Figure 3: CT at T12 level demonstrating similar features with contrast layering in the inferior vena cava, the inferior aspect of the left extrapleural haematoma still visible, contrast filled venous structures in the dependent erector spinae muscles, the sinusoidal opacification of the posterior aspect of the liver and a contrast filled right portal vein (arrow).

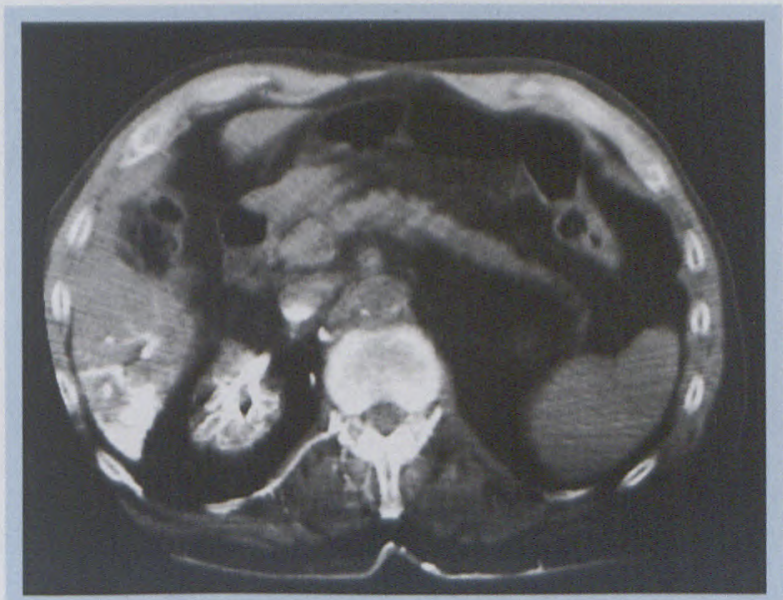

Figure 4: CT image at L1-L2 disc level demonstrating contrast layering in the inferior vena cava, flattening of the aorta from front to back, and very dense opacification of the right renal veins with an appearance similar to that of a diagnostic contrast renal venogram. fashion. The parenchyma of the right kidney was not opacified in the same manner, possibly as the renal parenchyma is of firmer consistency.

The contrast medium layering in the suprarenal inferior vena cava was remarkable, but little filling of the infrarenal vena cava was present. All veins and venous channels appeared to be dilated, but the aorta assumed a 'collapsed' configuration, being wider from side to side (Figure 4).

The patient died during the CT examination, and the diagnosis of aortic rupture was confirmed at a subsequent post-mortem examination. The site of rupture was $9 \mathrm{~cm}$ inferior to the left subclavian artery, and the aorta was markedly atherosclerotic. Two litres of left-sided posterior extrapleural blood was found; the pleura was bowed anteriorly in convex fashion. A further litre of intrapleural blood was found. The left lung was compressed and pale and weighed $340 \mathrm{~g}$, and the right lung was rusty in colour, oedematous and weighed $760 \mathrm{~g}$. There were signs of cardiomegaly and coronary atherosclerosis.

The above case presentation includes some unusual features, with death during CT being an unusual event and documented thus in pictorial form. It is also worthy of note that a large extrapleural haemothorax is unusual following traumatic aortic rupture, with the apical cap sign and right paraspinal line displacement occurring in $10 \%$, whereas a left haemothorax occurred in $16 \%$ of patients. ${ }^{1}$ At post-mortem examination the distinction between extrapleural and pleural haemothorax is not usually made. The CT in the above case report was performed as an emergency procedure, but during the examination it was decided that the patient would not be considered for resuscitation as a pre-existent diagnosis of Alzheimer's disease had been communicated at that stage.

\section{Reference}

1. Stark P. Traumatic rupture of the thoracic aorta; a review. CRC Critical Review in Diagnostic Imaging $1984 ; 21(3): 229-255$ 